\title{
THE ROLE OF IMMUNOHISTOCHEMISTRY IN THE EVALUATION OF UNDIFFERENTIATED GASTRIC CANCER
}

\author{
MAYADA I. YALDA, ALAA H. RAZIQ and BASHAR HASSAWI \\ College of Medicine, University of Duhok, Kurdistan Region-iraq
}

(Received: July 18, 2017; Accepted for publication: December 28, 2017)

\begin{abstract}
Background: With the advance in medical practices, gastric cancer remains one of the deadly diseases with poor prognosis. Appropriate diagnosis of the histological gastric cancer type may improve the treatment and the prognosis. The objective of this study is to apply immunohistochemical (IHC) markers for the diagnosis of undifferentiated gastric malignancies. Among 126 patients diagnosed as cases with malignant gastric tumors, from September 2008 to September 2013, 55 cases were assigned as undifferentiated tumors and were subjected to IHC evaluation by application of many IHC markers and special stains for further categorization; Results: After the application of different IHC markers and special stains, the 55 cases which were assigned as undifferentiated revealed to be carcinoma (37 cases), lymphoma ( 9 cases), gastrointestinal stromal tumors (GIST) (7 cases) and leiomyosarcoma and neuroendocrine tumor one case for each. IHC findings had changed the primary diagnoses based on morphological data in 4 instances; two cases were thought to be signet ring carcinoma and proved by IHC to be lymphomas and other two cases were thought to be poorly differentiated adenocarcinoma and proved to be GIST. Therefore; IHC is a valuable tool for the diagnosis of undifferentiated malignant gastric tumors.
\end{abstract}

KEY WORDS: Immunohistochemistry, Undifferentiated, Gastric, Cancer.

\section{INTRODUCTION}

$\mathbf{S}^{\mathrm{s}}$ tomach cancer is the fourth most common cancer in 2007 and the fifth in 2012 worldwide $(1,2)$. In Asia gastric adenocarcinoma (GA) is the second most common cancer.

Histologically, adenocarcinoma constitutes 90$95 \%$ of all gastric malignancies, followed by lymphomas (1-5\%), GIST (2\%), carcinoids (1\%), adenoacanthomas (1\%), and squamous cell carcinomas $(1 \%)(2,3)$.

According to the World Health Organization (WHO) and the Japanese classification of gastric tumors, the five predominant histological types of GA are: tubular adenocarcinoma, papillary adenocarcinoma, poorly differentiated adenocarcinoma, signet ring cell carcinoma (SRC) and mucinous adenocarcinoma (MAC) as shown in table $1^{(2,4)}$. Undifferentiated-type $\mathrm{GA}$ in general has a worst prognosis. The innate characteristics and prognosis of MAC and SRC have been studied $(5,6)$. However, the results of those studies are still debated. The tubular type GA shows variable expression of CK7, CK20, CDX-2, MUC1, and MUC5AC (7-9). Over 70\% of cases of the diffuse type GA are positive for CDX-2, CK7, HepPar-1and, variable expression of CK20, MUC2 and MUC5AC, but negative for MUC1 and E- cadherin $(10,11)$. Cases of poorly differentiated adenocarcinoma with prominent lymphoplasmacytic stroma may also be positive for $\operatorname{EBV}(12,13)$.

The vast majority of GISTs show a diffuse cytoplasmic staining with membranous accentuation of CD117 (KIT) ${ }^{(14)}$. The mucosaassociated lymphoid tissue (MALT) is the most common type of lymphoma to occur in the stomach (15). The lymphoma cells are B- cells and infiltrate the marginal zone around the preserved follicles. Tumor cells are positive for CD20, CD79a and Pax-5 but negative for CD5, CD10, and CD23 


\begin{tabular}{ll}
\hline \multirow{2}{*}{ Table (1): Histological classification of gastric cancer } \\
\hline \multirow{2}{*}{ Adenocarcinoma } & $\begin{array}{l}\text { Tubular adenocarcinoma (tub) } \\
\text { Papillarv adenocarcinoma (pap) }\end{array}$ \\
\cline { 2 - 2 } $\begin{array}{ll}\text { Poorly differentiated } \\
\text { adenocarcinoma (por) }\end{array}$ \\
\cline { 2 - 2 } $\begin{array}{ll}\text { Signet-ring cell carcinoma } \\
\text { Lymphomas }\end{array}$ & MALToma adenocarcinoma \\
\hline Mesenchymal & Other B and T lymphomas \\
\cline { 2 - 2 } & Leiomyosarcoma \\
\hline Carcinoids & \\
\hline Adenoacanthomas & \\
\hline Squamous cell carcinomas \\
\hline
\end{tabular}

\section{Aim of the study:}

To evaluate the role of IHC in ascertaining the histogenic origin of undifferentiated malignant gastric tumors which will affect the therapeutic and prognostic implications.

\section{PATIENTS AND METHODS}

This study included cases of gastric cancer diagnosed and reviewed histopathologically during a period of five years, from September 2008 to September 2013 in the central lab of Duhok-Iraq. The paraffin embedded blocks (PEBs) of biopsies from patients whom were diagnosed as having undifferentiated gastric malignancies (55 cases out of 126 gastric cancer cases) were selected to perform the immunehistochemical staining protocol according to the Avidin Biotin Complex (ABC) detection system $^{(17)}$. Sections from the PEBs where obtained in a 4 microns thickness and placed on positively charged slides together with adjacent parallel control sections which were processed with each set of staining for the IHC. Primary and secondary antibody kits were used, provided by the DAKO (an American company for Technologies), detected with the Envision+ system that employs peroxidase-labeled polymer conjugated to anti-mouse immunoglobulin antibodies. Immune complexes were identified by using peroxidase reaction with $\mathrm{DAB}+$ as chromogen (Envision+ detection system, K4006, Dako Corp, Carpinteria, CA). Different immunohistochemical markers have been used for different cases to identify the type of tumors as seen in table 2.

The selection of these combinations based mainly on preliminary histopathological findings using the few distinguishing microscopic characteristics. The results of panel 3 depend on the positive and negative combination.

\section{RESULTS}

During a period of five years there were 126 cases of gastric malignancy. The mean age of the patients was $54.3( \pm 14.36 \mathrm{SD})$ years. The commonest type of gastric malignancy was adenocarcinoma (71 cases)and representing 56.4\% of the cases. Out of these 126 cases $55(43.6 \%)$ were assigned as undifferentiated malignancy and subjected to IHC evaluation and special stains for their categorization. The mean age of these patients with undifferentiated malignancies was $60( \pm 13.0 \mathrm{SD})$ years. After the use of many IHC markers in different panels and special stains, the categories of malignancy were established. As shown in Table 3, the commonest types of gastric malignancies (in descending order) were carcinomas, lymphomas, GIST and the least were smooth muscle and neuroendocrine tumors (Table 3). IHC findings had changed the primary diagnoses based on morphological data in 4 instances, two were thought to be signet ring carcinoma and proved to be lymphomas and two were thought to be poorly differentiated adenocarcinoma and proved to be GIST.

The age of the 55 cases ranged from 25 to 93 years, with the youngest patient ( 25 year old female) diagnosed as undifferentiated carcinoma and the oldest patient (93 years old male) with GIST. The male to female ratio was about 1:1 (28 males and 27 females) as seen in table 4 . 
Table(2): Panels of IHC markers used for different types of malignancy

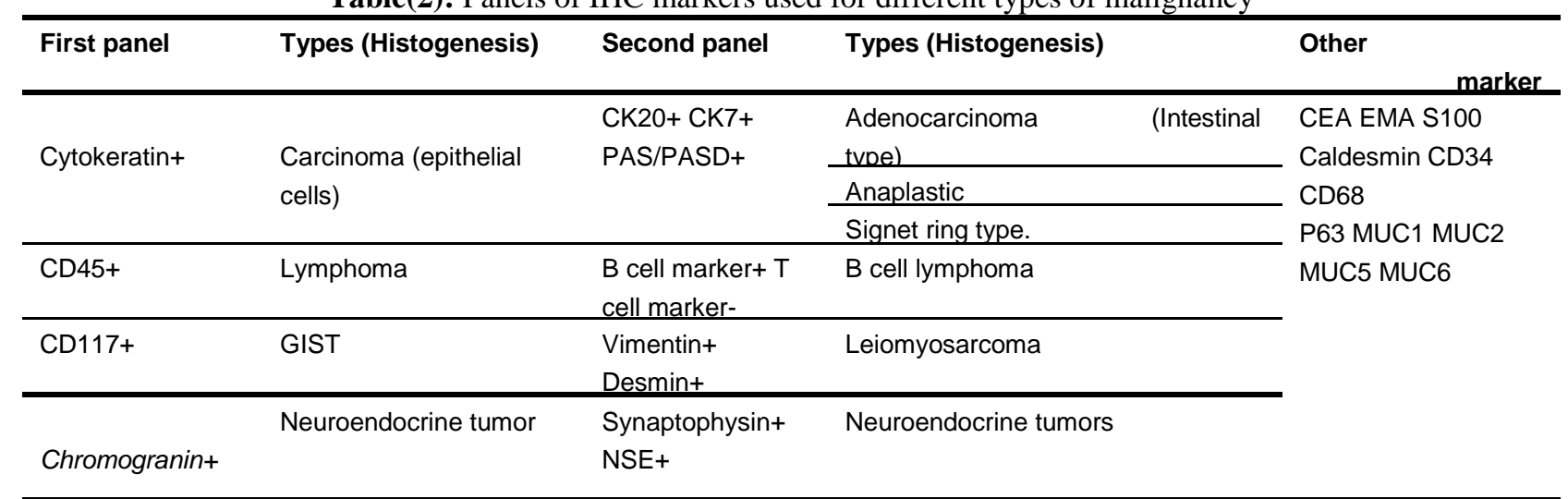

CD stands for Cluster of Differentiation, CK stands for Cytokeratin, PAS/PASD: Periodic acid-Schiff/ Periodic acid-Schiff diastase, NSE: Neuron- Specific Enolase, CEA: Carcino-Embryonic Antigen, EMA: Epithelial Membrane Antigen, P63: Protein 63, and the MUC stands for Mucin.

Table (3): Types of undifferentiated gastric cancer according to the IHC results

\begin{tabular}{lll}
\hline Type & Number & Percentage \\
\hline Carcinoma & $37(67.27 \%)$ & 67.27 \\
\hline Lymphoma & $9(16.36 \%)$ & 16.36 \\
\hline GIST & $7(12.73 \%)$ & 12.73 \\
\hline Leiomyosarcoma & $1(1.82 \%)$ & 1.82 \\
\hline Neuroendocrine tumor & $1(1.82 \%)$ & 1.82 \\
\hline Total & $55(100 \%)$ & $100 \%$ \\
\hline
\end{tabular}

Table (4): The frequency of undiefferentiated malignancy according to their histogenesis, age and gender.

\begin{tabular}{|c|c|c|c|}
\hline Types of undifferentiated malignancy after application of IHC & No. $(\%)$ & Mean age & ${ }^{\star} \mathrm{M}: \mathrm{F}$ ratio \\
\hline Carcinoma & $37(67.27 \%)$ & 52 & $1: 1$ \\
\hline Lymphoma & $9(16.36 \%)$ & 54 & $1: 2$ \\
\hline GIST & $7(12.73 \%)$ & 65.5 & 2.5:1 \\
\hline Leiomyosarcoma & $1(1.82 \%)$ & 55 & - \\
\hline Neuroendocrine tumor & $1(1.82 \%)$ & 57 & - \\
\hline Total & $55(100 \%)$ & 60 & $1: 1$ \\
\hline
\end{tabular}

* Male to female ratio

\section{DISCUSSION}

Although the incidence of gastric cancer has been declined in industrialized countries (from 774,000 in 1990 to 700,000 in 2012), it remains the third leading cause of cancer death ${ }^{(18,19)}$ and the five- year survival rate is less than 10 percent ${ }^{(20,21)}$. The undifferentiated-type gastric adenocarcinomas have a worse prognosis and carry even a less five-year survival rates.

In this study we tried to identify the different histological types of undifferentiated gastric cancers, taking in consideration that the treatment for stomach cancer depends on the histopathological type and may include surgery $^{(22)}$, chemotherapy, radiation ${ }^{(23)}$, and the new biological therapy $(20,24)$

In agreement with the findings all over the word, adenocarcinoma was the commonest type of gastric cancer in our research, but the undifferentiated type was higher than that reported in other studies ${ }^{(2-4)}$, probably because they $\mathrm{did}$ the classification after application of IHC analysis. However, the mean age of the undifferentiated malignancies in our study was higher than that of adenocarcinoma, while many other researches found that higher prevalence of "diffuse and undifferentiated types" is found in young patients, which may exhibit distinct disease characteristics and typically a worse outcome $(25,26)$. According to a population-based study of gastric cancer, a significant impact of age on survival was found 
only in patients with stage IV disease ${ }^{(27)}$. Other studies demonstrated that, when matched for stage, younger patients did not have worse outcomes $(28)$.

Furthermore the male to female ratio was nearly equal in this study, while Brenner $\mathrm{H}$ et al, stated that "As compared to women, men are twice as likely to develop and die from gastric cancer, in the US"(29). Although this may represent varying environmental exposures between genders, studies demonstrate that menstrual factors such as age of menopause and years of fertility are associated with gastritc cancer incidence $^{(30)}$.

These results of high undifferentiated cancers, age and gender, may suggest different genetics of gastric cancer in our society; therefore genetic analysis is suggested for DNA sequences and special gene

expression of gastric cancers. Finally we should emphasis on the importance of IHC analysis for cases of gastric cancer with uncertain histological findings.

\section{CONCLUSIONS}

This study demonstrates the importance of IHC application for undifferentiated cancer which benefits in the establishment of the histological type and which may also change the morphology based diagnosis.

\section{REFERENCES}

- WHO. "Are the number of cancer cases increasing or decreasing in the world?" WHO Online Q\&A. 1 April 2008. Retrieved 2009-05- 11.

- World Cancer Report "Chapter 1.1" 2014. World Health Organization. 2014.

- Lambert R, Guilloux A, Oshima A, Pompe-Kirn V, Bray F, Park M, et al. Incidence and mortality from stomach cancer in Japan, Slovenia and the USA. Int J Cancer 2002; 97:811-818.

- The International Gastric Cancer Association and the Japanese Gastric Cancer Association. Japanese classification of gastric carcinoma: 3rd English edition. Gastric Cancer 2011, 14:101- 112.

- Fang WL, Wu CW, Lo SS, Chen JH, Hsieh MC, Shen $\mathrm{KH}$, et al. Mucin-producing gastric cancer:

clinicopathological difference between signet ring cell carcinoma and mucinous carcinoma. Hepato-gastro-enterology 2009, 56:1227-1231.
- Zhang M, Zhu G, Zhang H, Gao H, Xue Y: Clinicopathologic features of gastric carcinoma with signet ring cell histology. J Gastrointest Surg 2010, 14:601 606.

- Taniere P, Borghi-Scoazec G, Saurin JC, LombardBohas C, Boulez J, Berger F et al. Cytokeratin expression in adenocarcinomas of the esophagogastric junction: a comparative study of adenocarcinomas of the distal esophagus and of the proximal stomach. Am J Surg Pathol 2002;26:1213-1221.

- Flucke U, Steinborn E, Dries V, Mönig SP, Schneider PM, Thiele J, et al. Immunoreactivity of cytokeratins (CK7, CK20) and mucin peptide core antigens (MUC1, MUC2,

MUC5AC) in adenocarcinomas, normal and metaplastic tissues of the distal oesophagus, oesophago-gastric junction and proximal stomach. Histopathology 2003;43:127-134.

- Gulmann C, Counihan I, Grace A, Patchett S, Leen E, Leader M, et al. Cytokeratin 7/20 and mucin expression patterns in oesophageal, cardia and distal gastric adenocarcinomas. Histopathology 2003;43:453- 461.

- Lau SK, Weiss LM, Chu PG. Differential expression of MUC1, MUC2, and MUC5AC in carcinomas of various sites: an immunohistochemical study. Am J Clin Pathol 2004;122:61-69.

- Goldstein NS, Long A, Kuan SF, Hart J. Colon signet ring cell adenocarcinoma: immunohistochemical characterization and comparison with gastric and typical colon adenocarcinomas. Appl Immunohistochem Mol Morphol 2000;8:183188.

- Shibata D, Tokunaga M, Uemura Y, Sato E, Tanaka S, Weiss LM. Association of Epstein-Barr virus with undifferentiated gastric carcinomas with intense lymphoid infiltration. Lymphoepithelioma-like carcinoma. Am J Pathol 1991;139:469-474.

- Shibata D, Weiss LM. Epstein-Barr virus- associated gastric adenocarcinoma. Am J Pathol 1992;140:769-774.

- Miettinen M, Lasota J. Gastrointestinal stromal tumors: pathology and prognosis at different sites. Semin Diagn Pathol 2006;23:70-83.

- Doglioni C, Wotherspoon AC, Moschini A, de Boni $\mathrm{M}$, Isaacson PG. High incidence of primary gastric lymphoma in northeastern Italy. Lancet 1992; 339:834-835.

- Lai R, Weiss LM, Chang KL, Arber DA. Frequency of CD43 expression in non-Hodgkin lymphoma. A survey of 742 cases and further characterization of rare $\mathrm{CD}^{+} 3^{+}$follicular lymphomas. Am J Clin Pathol 1999;111:488- 
494.

- Mangham DC, Bradwell AR, Isaacson PG. MICA-a highly sensitive and avidin-free immunehistochemical detection system. dv Anat Pathol. 2000; Nov; 7 (6):360-364.

- Lozano R, Naghavi M, Foreman K, Lim S, Shibuya $\mathrm{K}$, Aboyans V, et al. Global and regional mortality from 235 causes of death for 20 age groups in 1990 and 2010: a systematic analysis for the Global Burden of Disease Study 2010. Lancet 2012; 2095-2128.

- World Health Organization "PRESS RELEASE" Global battle against cancer won't be won with treatment alone Effective prevention measures urgently needed to prevent cancer crisis. 3 February 2014. Retrieved 14 March2014.

- American Cancer Society. Cancer Facts \& Figures 2015. Atlanta, Ga: American Cancer Society; 2015.

- Orditura M, Galizia G, Sforza V, Gambardella V, Fabozzi A, Laterza MM, et al. Treatment of gastric cancer. World Journal of Gastroenterology 2014; 20 (7); 1635-1649.

- Chen K, Xu X.W, Zhang R-C, Mou YP, Pan Y, Wu $D$, et al. Systematic review and meta-analysis of laparoscopy-assisted and open total gastrectomy for gastric cancer. World J Gastroenterol 2013; 19 (32): 5365-5376.

- Pretz JL, Jennifer Y. Harvey J. Mamon J, Lisa A. Chemoradiation Therapy: Localized Esophageal, Gastric, and Pancreatic Cancer.
Surgical Oncology Clinics of North America. 2011; 22 (3): 511-524.

- Judith M-J, Heather J Au, Michael Bl. Critical appraisal of trastuzumab in treatment of advanced stomach cancer. Cancer Management and Research 2011 (3): 57-64.

- Kong X, Wang JL, Chen HM, Fang JY. Comparison of the clinicopathological characteristics of young and elderly patients with gastric carcinoma: a meta-analysis. J Surg Oncol 2012;106: 346-352.

- Saito H, Takaya S, Fukumoto Y, Osaki T, Tatebe S, Ikeguchi M. Clinicopathologic characteristics and prognosis of gastric cancer in young patients. Yonago Acta Med 2012; 55:57-61.

- Al-Refaie WB, Pisters PW, Chang GJ. Gastric adenocarcinoma in young patients: A population- based appraisal. J Clin Oncol. 2007; 25:4547.

- Santoro R, Carboni F, Lepiane P, Ettorre GM, Santoro E. Clinicopathological features and prognosis of gastric cancer in young European adults. Br J Surg 2007; 94:737-742.

- Brenner H, Rothenbacher D, Arndt V. Epidemiology of stomach cancer. Methods Mol Biol. 2009; 472:467-477.

- Freedman ND, Chow WH, Gao YT, Shu XO, Ji BT, Yang G, et al. Menstrual and reproductive factors and gastric cancer risk in a large prospective study of women. Gut. 2007; 56:1671-1677. 